\title{
Ultrasound Evaluation of an Abdominal Aortic Fluid-Structure Interaction Model
}

\section{Traberg, Marie Sand; Jensen, Jørgen Arendt}

\section{Published in:}

Proceedings of IEEE International Ultrasonics Symposium,

Link to article, DOI:

10.1109/ULTSYM.2014.0571

Publication date:

2014

Document Version

Early version, also known as pre-print

Link back to DTU Orbit

Citation (APA):

Traberg, M. S., \& Jensen, J. A. (2014). Ultrasound Evaluation of an Abdominal Aortic Fluid-Structure Interaction Model. In Proceedings of IEEE International Ultrasonics Symposium, (pp. 2292-2295). IEEE.

https://doi.org/10.1109/ULTSYM.2014.0571

\section{General rights}

Copyright and moral rights for the publications made accessible in the public portal are retained by the authors and/or other copyright owners and it is a condition of accessing publications that users recognise and abide by the legal requirements associated with these rights.

- Users may download and print one copy of any publication from the public portal for the purpose of private study or research.

- You may not further distribute the material or use it for any profit-making activity or commercial gain

- You may freely distribute the URL identifying the publication in the public portal 
Paper presented at the IEEE International Ultrasonics Symposium, Chicago, Il., USA, 2014:

\section{Ultrasound Evaluation of an Abdominal Aortic Fluid-Structure Interaction Model}

Marie Sand Traberg and Jørgen Arendt Jensen

Center for Fast Ultrasound Imaging,

Biomedical Engineering group, Department of Electrical Engineering, Bldg. 349, Technical University of Denmark, DK-2800 Kgs. Lyngby, Denmark

To be published in Proceedings of IEEE International Ultrasonics Symposium, Chicago, Il., USA, 2014. 


\title{
Ultrasound Evaluation of an Abdominal Aortic Fluid-Structure Interaction Model
}

\author{
Marie Sand Traberg and Jørgen Arendt Jensen \\ Center for Fast Ultrasound Imaging, Department of Electrical Engineering, \\ Technical University of Denmark, DK-2800 Lyngby, Denmark
}

\begin{abstract}
Ultrasound measurements are used for evaluating biomechanics of the abdominal aorta (AA) predicted by a fluidstructure interaction (FSI) simulation model. FSI simulation models describe the complete arterial physiology by quantifying the mechanical response in the vessel wall caused by the percolating pulsating blood. But the predictability of FSI models needs validation for these to be usable for diagnostic purposes. Ultrasound measurements are suitable for such an evaluation as the wall displacement can be measured in vivo and compared to the wall displacement simulated in the FSI model. Spectral Doppler velocity data from 3 healthy male volunteers were used to construct inlet profiles for the FSI model. Simultaneously, wall movement was tracked and used for comparison to FSI model results. Ultrasound data were acquired using a scanner equipped with a research interface. The wall displacement was estimated by time shift estimation obtained from cross-correlation of signals to a fixed reference. The FSI model was constructed as a 2D axissymmetric pipe with lumen diameter predicted by B-mode images from each volunteer. Visual comparison of wall displacement over 1 cardiac cycle show agreement except for 1 volunteer (Male, 23 yrs.). The magnitude of the displacement in simulation, $u_{\mathrm{fsi}}$, and in vivo, $\mathrm{u}_{\mathrm{iv}}$, is within the same order of magnitude for the young $\left(u_{i v}=1.48 \mathrm{~mm}, u_{\mathrm{fsi}}=1.12 \mathrm{~mm}\right)$ and middle-aged volunteer $\left(u_{i v}=0.783 \mathrm{~mm}, u_{f s i}=1.31 \mathrm{~mm}\right)$. For the elderly volunteer the simulated displacement $\left(u_{\mathrm{fsi}}=0.975 \cdot 10^{-3} \mathrm{~mm}\right)$ is much smaller compared to in vivo $\left(\mathrm{u}_{\mathrm{iv}}=0.979 \mathrm{~mm}\right)$. In conclusion, the FSI model predicts a much stiffer AA wall compared to measured displacements for the elderly volunteer. From the visual comparison in vivo wall motion is captured in the FSI model for 2 of the 3 volunteers.
\end{abstract}

\section{INTRODUCTION}

Atherosclerosis and aneurysms are speculated to be caused by an imbalance in the vascular adaptation to diverse mechanobiological stimuli [1], [2]. The abdominal aorta (AA) is location for development of both atherosclerosis and aneurysms. It is therefore interesting to study the AA wall in a computational simulation environment to gain knowledge about the onset of mechanical events which can lead to pathologies. Fluidstructure interaction (FSI) simulation models combine computational fluid dynamics and solid mechanical modeling using finite element analysis. These models are computationally heavy but have the advantage of including the complete arterial physiology by quantifying the mechanical response in the vessel wall caused by the percolating pulsating blood. Working with FSI models it is worth considering whether the chosen model is comparable to the in vivo situation. Therefore, the objective of this work is to compare in vivo AA wall displacement to simulated wall displacement obtained from a FSI model.

\section{METHODS}

The use of ultrasound scan was twofold. Blood flow data obtained by spectral Doppler was used to construct inlet profiles for the FSI model. Simultaneously, wall movement was tracked and used for comparison to FSI model results.

\section{A. Acquisition of ultrasound data}

Spectral data were acquired using a convex array transducer connected to a 2202 ProFocus scanner (BK Medical, Herlev, Denmark) equipped with a UA2227 research interface [3]. Post-processing was performed in Matlab. For this work three male volunteers aged 23, 53 and 76 years (yrs) respectively, were scanned with ultrasound. Each volunteer was scanned several times, and each scan sequence lasted five seconds. Details of the scanning procedure is described in [4].

\section{B. Reconstruction of inlet velocity profiles}

Inlet profiles were obtained by harmonic decomposition of the measured average flow velocity and using the WomersleyEvans model [5], [6] to reconstruct smooth the profiles for the finite element based FSI model. A detailed description of velocity profile reconstruction can be found in [4].

\section{Estimation of wall displacement}

The wall displacement can be derived directly from the raw RF spectral flow data by removing the stationary echo canceling filter and apply the time shift estimation approach. The displacement is determined by,

$$
\Delta z=\frac{t_{s} c}{2} \sin (\theta)
$$

where $\Delta z$ is the displacement, $t_{s}$ is the time shift, $c=1540$ $\mathrm{m} / \mathrm{s}$ is the speed of sound in soft tissue, and $\theta$ is the beamto-flow angle. Angle correction of the displacement data is needed as the data acquisition required manual alignment of the flow direction in the range gate used to obtain spectral flow data, see Fig. 2.

The time shift was estimated using the cross-correlation between consecutive received signals. For each estimate 10 lines of flow data were used. The peaks of the cross-correlation functions were found by interpolation around the lag, $n_{m}$, of the peak [7], 



Fig. 1. Velocity variation in the center of the AA for each volunteer.

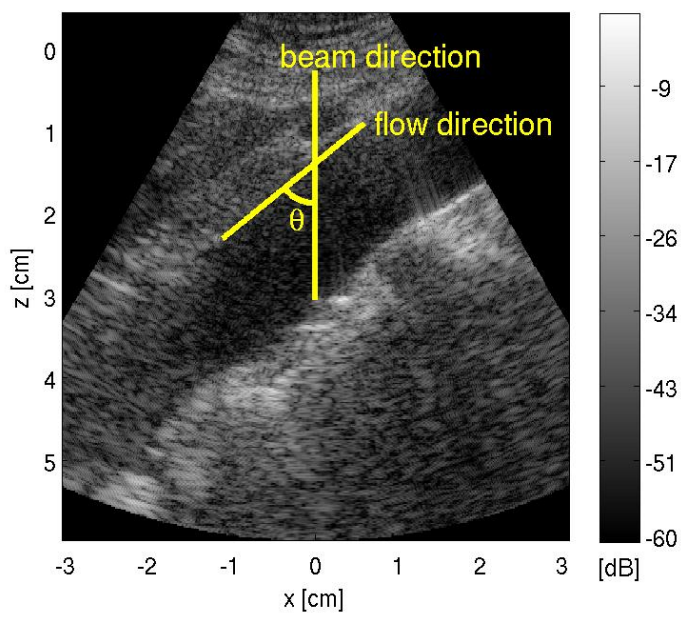

Fig. 2. B-mode image example from scanning of the AA with illustration of the beam-to-flow angle, $\theta$.

$n_{i n t}=n_{m}-\frac{\hat{R}_{12 d}\left(n_{m}+1\right)-\hat{R}_{12 d}\left(n_{m}-1\right)}{2\left(\hat{R}_{12 d}\left(n_{m}+1\right)-2 \hat{R}_{12 d}\left(n_{m}\right)+\hat{R}_{12 d}\left(n_{m}-1\right)\right)}$

where $n_{\text {int }}$ is the lag of the interpolated peak and $\hat{R}_{12 d}$ is the value of the cross-correlation function. Parameters for the time shift estimation are shown in Table I.

\section{Fluid-structure interaction model}

The FSI model was constructed in COMSOL Multiphysics v4.4 (COMSOL AB, Stockholm, Sweden) using the FluidStucture Interaction Interface.

1) Fluid domain: The constitutive framework for the fluid domain of the FSI model simulations was the Navier-Stokes equation assuming that blood is an incompressible isotropic Newtonian fluid,
TABLE I

EXAMPLE OF PARAMETERS FOR THE AXIAL DISPLACEMENT ESTIMATION

\begin{tabular}{lcl}
\hline \hline Parameter & Symbol & Value \\
\hline Transducer frequency & $f_{0}$ & $3.0 \mathrm{MHz}$ \\
Sampling frequency & $f_{s}$ & $12 \mathrm{MHz}$ \\
Pulse repetition frequency & $f_{\text {prf }}$ & $2012 \mathrm{~Hz}$ \\
Range gate size & $l_{g}$ & $0.64 \mathrm{~mm}$ \\
Lines for one estimate & $N_{c}$ & $10-12$ \\
\hline \hline
\end{tabular}

$$
\rho_{\text {blood }}\left(\frac{\partial \vec{v}}{\partial t}+(\vec{v} \cdot \nabla) \vec{v}\right)=-\nabla p+\mu_{\text {blood }} \nabla^{2} \vec{v}+\rho_{\text {blood }} \vec{g}
$$

where $\vec{v}$ is the velocity field, $\nabla$ is the vector differential operator, $\nabla p$ is the pressure gradient, $\nabla^{2}$ is the Laplacian, and $\vec{g}$ is gravity. The density of blood was set to $\rho_{\text {blood }}=1,060$ $\mathrm{kg} / \mathrm{m}^{2}$ and the viscosity of blood was set to $\mu_{\text {blood }}=3.5$ $\mathrm{mPa} \cdot \mathrm{s}$. The inlet condition for the fluid domain was governed by the reconstructed subject-specific flow profiles, see Fig. 1 , described in II-B. The outlet condition was a $0 \mathrm{mmHg}$ uniform pressure. The boundary condition for the wall of the fluid domain was dictated by the fluid-structure interaction as described below.

2) Solid domain: The AA wall material was hyperelastic nonlinear and anisotropic with age-matched material parameters [8], [9]. The aortic wall material properties are represented in a strain energy function [10], [11]. The specific form of the strain energy function builds on histological observations and it is composed of a isotropic elastin dominated amorphous matrix re-enforced by four families of collagen fibers identified by their orientation. An illustration of the concept behind the strain energy function is shown in Fig. 4. The material model represented by the strain energy function is fitted with agematched parameters adapted from [9]. The simulation model use finite element analysis to determine the displacement 


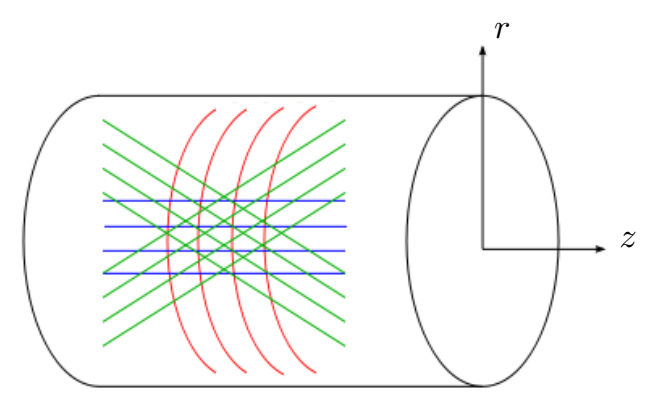

Fig. 3. Illustration of the principle behind the strain energy function with four families of collagen fibers. Red are circumferential (hoop) oriented collagen fibers, blue are axial oriented collagen fibers, and green are two families of diagonal oriented collagen fibers.

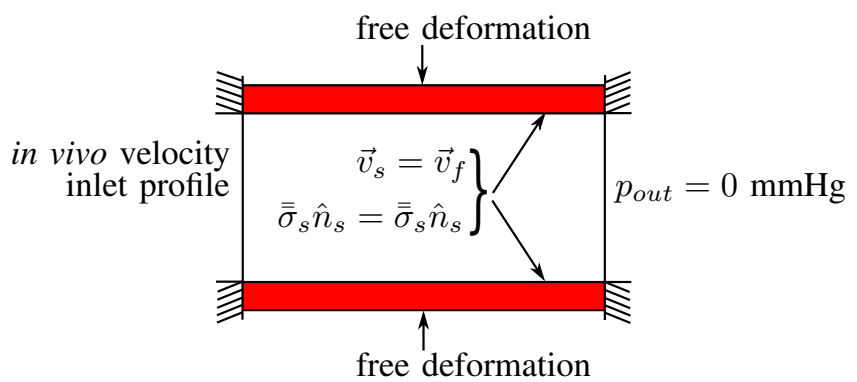

Fig. 4. Illustration of the applied boundary conditions in the FSI model The subscript $f$ is associated with the fluid domain and the subscript $s$ is associated with the solid domain.

experienced by the structure due to the applied flow and boundary conditions. The displacement, $\vec{u}$ is determined as,

$$
\vec{u}(\vec{X}, t)=\vec{x}(\vec{X}, t)-\vec{X},
$$

where $\vec{X}$ is material point position in reference frame, and $\vec{x}$ is current material point position. The solid domain of the FSI model was fixed in both ends simulating the aortic tethering. At the inner wall the deformation was dictated by fluid-structure interaction, see Sec. II-D4.

3) Model geometry: For simplicity the AA wall in all cases was assumed to be a two-dimensional axis-symmetric circular cylindrical pipe. The length of the pipe was $100 \mathrm{~mm}$, and the diameter was determined the B-mode ultrasound image for each volunteer. The AA wall had a thickness of $1.5 \mathrm{~mm}$ in the reference configuration.

4) Fluid-structure interaction simulation: Blood velocity and pressure fields are influenced by the deformation of the AA wall. Usually Eq. (3) is solved on a fixed Eulerian reference frame, but to account for the deforming AA wall Eq. (3) must be solved in a moving reference frame. Here the arbitrary Lagrangian-Eulerian (ALE) formulation is applied to quantify the fluid-structure interaction. This formulation has been used by several researchers [12], [13]. In brief, the Eq. (3) is written on a moving reference frame to allow motion of the fluid-structure interface. Simulation of FSI relies on kinematic and dynamic compatibility conditions between the two domains [12]:
TABLE II

DISPLACEMENT MAGNITUDES FOR THE 3 VOLUNTEERS.

\begin{tabular}{ccc}
\hline \hline & In vivo & Simulation \\
\hline \hline Male, 23 yrs. & $1.48 \mathrm{~mm}$ & $1.12 \mathrm{~mm}$ \\
Male, 53 yrs. & $0.783 \mathrm{~mm}$ & $1.31 \mathrm{~mm}$ \\
Male, 76 yrs. & $0.979 \mathrm{~mm}$ & $0.975 \cdot 10^{-3} \mathrm{~mm}$ \\
\hline \hline
\end{tabular}

1) The rate of change for the solid wall displacement acts as the moving wall for the fluid domain which ensures continuity of velocities.

2) The total force exerted by the fluid on the solid wall is the negative of the reaction force on the fluid which ensures continuity of forces.

The FSI couplings appear on the boundary between the fluid and the solid, and thus provides the boundary condition for the inner wall. The boundary conditions are illustrated in Fig. . When solving the FSI model, the simulation was run for 10 cycles repeating the inlet velocity variation 10 times. This was done to ensure stability of the solution and giving the pressure wave time enough to propagate down the AA. The computation time for the three different volunteers was 2,867 $\mathrm{s}, 2,238 \mathrm{~s}$ and $1691 \mathrm{~s}$ for the young volunteer ( $23 \mathrm{yrs})$, the middle-aged volunteer (53 yrs) and elderly volunteer ( $76 \mathrm{yrs}$ ) respectively.

\section{RESULTS}

In Fig. 6 the in vivo displacement along the ultrasound beam, i.e. change in AA radius, is compared to the radial displacement component in the FSI simulation for each volunteer. Positive displacement refers to expansion of the AA. By visual comparison it can be seen in Fig. 6a that the displacement over time is not captured by the FSI model for the youngest volunteer. However, the magnitude of the displacement is within the same order of magnitude, see Table II. The time-dependent displacement in Fig. $6 \mathrm{~b}$ show the same pattern for the in vivo measurement and simulated motion. So, the material model captures the motion in this case. Also, the magnitudes a within one order of magnitude, see Table II. For the elderly volunteer the magnitude of the displacement in the FSI model is three orders of magnitude smaller compared to in vivo measurements, see Table II and Fig. 6c. But in Fig. ?? the $y$-axis has been scaled, and comparing the time-dependent displacement from FSI simulations to in vivo, it is seen that the FSI model also in this case captures the motion.

\section{DisCUSSION AND CONCLUSION}

Crosetto et al. [14] show similar motion as displayed in Fig. $6 \mathrm{a}$ in the thoracic aorta of one healthy volunteer. They find that when applying a pressure in the inlet and a flux on the outlet of the fluid domain less physiological pressure waveforms are obtained in the distal part of the vessel. This is argued to be due to inappropriate pressure wave reflections at the outlet. This can be compensated by applying fluxes at both inlet and outlet. In this work, a velocity field is applied in 
a) Volunteer: Male, 23 yrs.

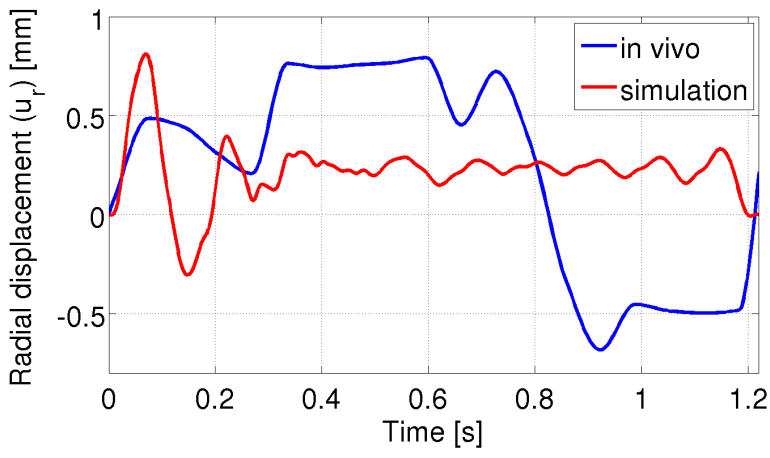

b) Volunteer: Male, 53 yrs.

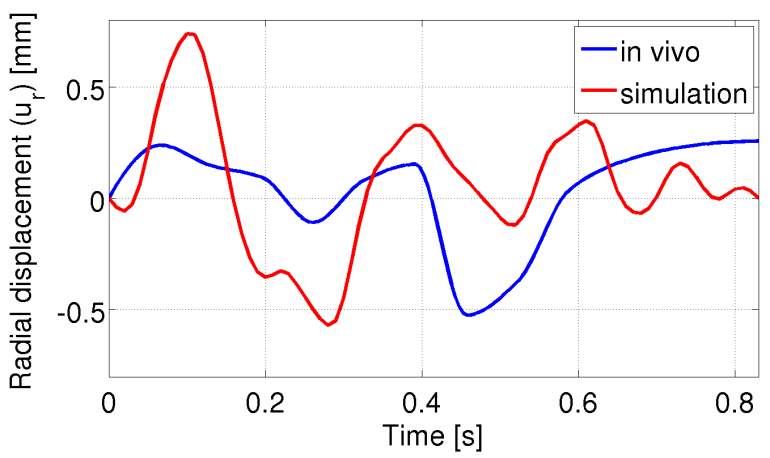

c) Volunteer: Male, 76 yrs.

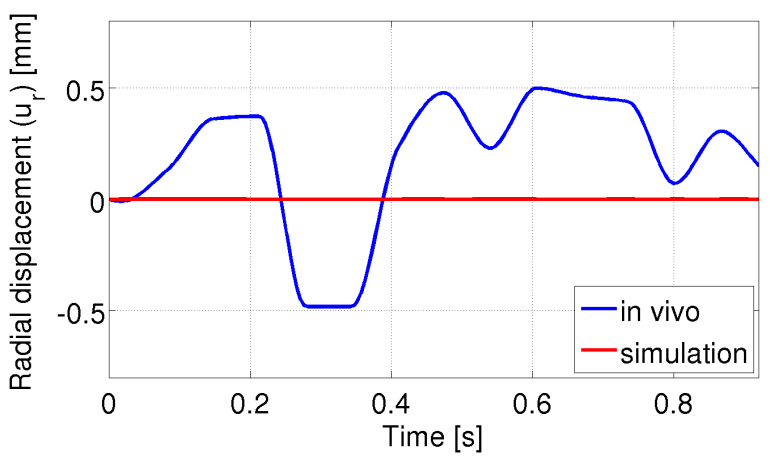

Fig. 5. Comparison of displacement in vivo and simulation for all three volunteers.

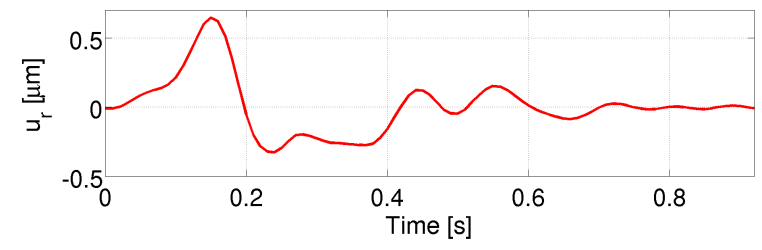

Fig. 6. Simulated time-dependent displacement for the elderly volunteer (Male, 76 yrs.).

the inlet and a homogeneous pressure is applied at the outlet. This can give rise to inappropriate pressure wave reflections in the vessel. This could explain the difference between the displacement variation in vivo and in simulation seen in Fig. $6 \mathrm{a}$ as the displacement is mainly given by the pressure change during the heart cycle. So, to make the FSI model presented here more realistic and comparable to in vivo measurements the effect of the boundary conditions on the fluid domain should be investigated further. Also, it is important to note that only three volunteers are included in the study, so the results should be interpreted with caution. Hence more cases are needed to perform a proper validation of the proposed FSI model. Other limitations of the FSI model are the fact that no axial pre-stess is included, and the reference position $(t=0)$ is assumed stress-free.

\section{ACKNOWLEDGMENT}

The authors would like to thank PhD, MD Mads Møller Pedersen for assistance with the ultrasound scans.

\section{REFERENCES}

[1] W. W. Nichols and M. F. O'Rourke, McDonald's Blood Flow in Arteries, Theoretical, Experimental and Clinical Principles. Philadelphia: Lea \& Febiger, 1990.

[2] A. Valentin, J. D. Humphrey, and G. A. Holzapfel, "A multi-layered computational model of coupled elastin degradation, vasoactive dysfunction, and collagenous stiffening in aortic aging," Ann. Biomed. Eng., vol. 39, no. 7, pp. 2027-2045, 2011.

[3] M. C. Hemmsen, S. I. Nikolov, M. M. Pedersen, M. J. Pihl, M. S. Enevoldsen, J. M. Hansen, and J. A. Jensen, "Implementation of a versatile research data acquisition system using a commercially available medical ultrasound scanner," IEEE Trans. Ultrason., Ferroelec., Freq. Contr., vol. 59, no. 7, pp. 1487-1499, 2012.

[4] M. S. Enevoldsen, M. M. Pedersen, M. C. Hemmsen, M. B. Nielsen, and J. A. Jensen, "Computational fluid dynamics using in vivo ultrasound blood flow measurements," in Proc. IEEE Ultrason. Symp., 2012, pp. $1596-1599$.

[5] J. R. Womersley, "Oscillatory motion of a viscous liquid in a thin-walled elastic tube. I: The linear approximation for long waves," Phil. Mag., vol. 46, pp. 199-221, 1955.

[6] D. H. Evans, "Some aspects of the relationship between instantaneous volumetric blood flow and continuous wave Doppler ultrasound recordings III," Ultrasound Med. Biol., vol. 8, pp. 617-623, 1982.

[7] J. A. Jensen, Estimation of Blood Velocities Using Ultrasound: A Signal Processing Approach. New York: Cambridge University Press, 1996.

[8] S. Baek, R. L. Gleason, K. R. Rajagopal, and J. D. Humphrey, "Theory of small on large: Potential utility in computations of fluid-solid interactions in arteries," Comput. Methods. Appl. M., vol. 196, pp. 3070-3078, June 2007.

[9] J. Ferruzzi, D. A. Vorp, and J. D. Humphrey, "On constitutive descriptors of the biaxial mechanical behaviour of human abdominal aorta and aneurysms," J. R. Soc. Interface, vol. 8, no. 56, pp. 435-450, March 2011.

[10] G. A. Holzapfel, T. C. Gasser, and R. W. Ogden, "A new constitutive framework for arterial wall mechanics and a comparative study of material models," J. Elasticity, vol. 61, pp. 1-48, 2000.

[11] P. D. Achille, S. Celi, F. D. Puccio, and P. Forte, "Anisotropic aaa: Computational comparison between four and two fiber family material models," J. Biomech., vol. 44, no. 13, pp. 2418-2426, September 2011.

[12] C. A. Taylor and C. A. Figueroa, "Patient-specific modeling of cardiovascular mechanics," Ann. Rev. Biomed. Eng., vol. 11, pp. 109-134, 2009.

[13] L. Taelman, J. Degroote, A. Swillens, J. Vierendeels, and P. Segers, "Fluid-structure interaction simulation of pulse propagation in arteries: Numerical pitfalls and hemodynamic impact of a local stiffening," Int. J Eng. Sci., vol. 77, pp. 1-13, 2014.

[14] P. Crosetto, P. Reymond, S. Deparis, D. Kontaxakis, N. Stergiopulos, and A. Quarteroni, "Fluid-structure interaction simulation of aortic blood flow," Computers and Fluids, vol. 43, pp. 46-57, 2011. 